\title{
Heavy-Ion-Induced Electronic Desorption of Gas from Metals
}

\author{
A. W. Molvik, ${ }^{1,2}$ H. Kollmus, ${ }^{3}$ E. Mahner, ${ }^{4}$ M. Kireeff Covo, ${ }^{1,2}$ M. C. Bellachioma, ${ }^{3}$ M. Bender, ${ }^{3}$ F. M. Bieniosek,,${ }^{1,5}$ \\ E. Hedlund, ${ }^{6}$ A. Krämer, ${ }^{3}$ J. Kwan, ${ }^{1,5}$ O. B. Malyshev, ${ }^{7}$ L. Prost,,${ }^{5,}$ P. A. Seidl,,${ }^{1,5}$ G. Westenskow, ${ }^{1,2}$ and L. Westerberg ${ }^{6}$ \\ ${ }^{1}$ Heavy-Ion Fusion Science Virtual National Laboratory, Berkeley, California 94720, USA \\ ${ }^{2}$ Lawrence Livermore National Laboratory, Livermore, California 94550, USA \\ ${ }^{3}$ GSI, Planckstrasse 1, 64291 Darmstadt, Germany \\ ${ }^{4}$ CERN, 1211 Geneva 23, Switzerland \\ ${ }^{5}$ Lawrence Berkeley National Laboratory, Berkeley, California 94720, USA \\ ${ }^{6}$ Uppsala University, 751 21, Uppsala, Sweden \\ ${ }^{7}$ CCLRC Daresbury Laboratory, Warrington, United Kingdom \\ (Received 6 November 2006; published 6 February 2007)
}

\begin{abstract}
During heavy-ion operation in several particle accelerators worldwide, dynamic pressure rises of orders of magnitude were triggered by lost beam ions that bombarded the vacuum chamber walls. This ioninduced molecular desorption, observed at CERN, GSI, and BNL, can seriously limit the ion beam lifetime and intensity of the accelerator. From dedicated test stand experiments we have discovered that heavy-ion-induced gas desorption scales with the electronic energy loss $\left(d E_{e} / d x\right)$ of the ions slowing down in matter; but it varies only little with the ion impact angle, unlike electronic sputtering.
\end{abstract}

PACS numbers: 41.75.Ak, 34.50.Dy, 79.20.Rf

Energetic ions incident on matter sputter target material and also desorb gas from the target surface. The sputter and desorption yields (number of sputtered or desorbed particles per ion impact) are known to be linked to the energy loss of the projectile inside the target. Two energy loss regimes, nuclear and electronic, have been known for decades. An example for potassium ions impacting onto stainless steel, calculated with the SRIM code [1], is shown in Fig. 1. Here for low projectile energies the nuclear energy loss dominates and for higher energies the electronic energy loss dominates the total energy loss. Sputter and desorption yield measurements from $\sim 1 \mu \mathrm{m}$ thick targets in the regime of electronic energy loss have shown that both scale with the electronic energy loss $\left(d E_{e} / d x\right)^{n}$ to the power of $n=1-3$. This was observed for targets of frozen gases [2-5], for sputtering from micron-thick coatings of protein [6,7], and for desorption of nitrogen from a conductor (carbon) by $6-13 \mathrm{MeV} / \mathrm{u}$ ions ( $\mathrm{u}$ is the nucleon mass) [8,9]. Electronic sputtering and desorption from $\sim 1 \mu \mathrm{m}$ thick targets is found to vary with the ion impact angle from normal, $\theta$, as $1 / \cos ^{m}(\theta)$ to the first or higher power of $m[5,10]$.

Our research was motivated by the copious gas desorption that results from lost heavy ions striking particle accelerator vacuum chambers leading to dynamic pressure rises which limit the beam intensity in a number of heavyion accelerators [11]. Related work dates back more than 30 years, when a vacuum instability in the Intersecting Storage Rings (ISR) at CERN was identified above a critical beam current [12]. Recent requirements for orders of magnitude increase in beam intensity have motivated our search for further understanding and mitigation mechanisms. In preparation for the heavy-ion program of the Large Hadron Collider at CERN, beam-loss induced molecular desorption was intensively studied in ultra-high- vacuum chambers at CERN's Heavy-Ion Accelerator (LINAC 3) [13] and the Super Proton Synchrotron (SPS) [14]. Large effective desorption yields of up to $2 \times$ $10^{4}$ molecules $/ \mathrm{Pb}^{53+}$ ion $(4.2 \mathrm{MeV} / \mathrm{u})$ and $3.7 \times$ $10^{4}$ molecules $/ \mathrm{In}^{49+}$ ion $(158 \mathrm{GeV} / \mathrm{u})$ were measured for ions impacting under various angles $\left[\theta=0^{\circ}\right.$ (perpendicular), $84.8^{\circ}, 89.2^{\circ}$ at LINAC 3 , and $88.3^{\circ}$ at the SPS] onto stainless steel samples which were chemically cleaned, $950{ }^{\circ} \mathrm{C}$ vacuum fired, and in situ baked at $300{ }^{\circ} \mathrm{C}$.

Similar yields of $1.1-2.7 \times 10^{4}$ molecules/ion were measured at the GSI Darmstadt SIS 18 for $8.9 \mathrm{MeV} / \mathrm{u}$ $\mathrm{U}^{28+}$ ions impacting near grazing incidence onto baked stainless steel vacuum chambers [15]. In a dedicated test stand (GSI-HLI) yields were 300 for $1.4 \mathrm{MeV} / \mathrm{u} \mathrm{Cr}^{7+}$ and 1900 for $1.4 \mathrm{MeV} / \mathrm{u} \mathrm{Pb}^{27+}$ perpendicularly impacting on stainless steel. In the High-Current Experiment (HCX) at Lawrence Berkeley National Laboratory (LBNL), gas de-

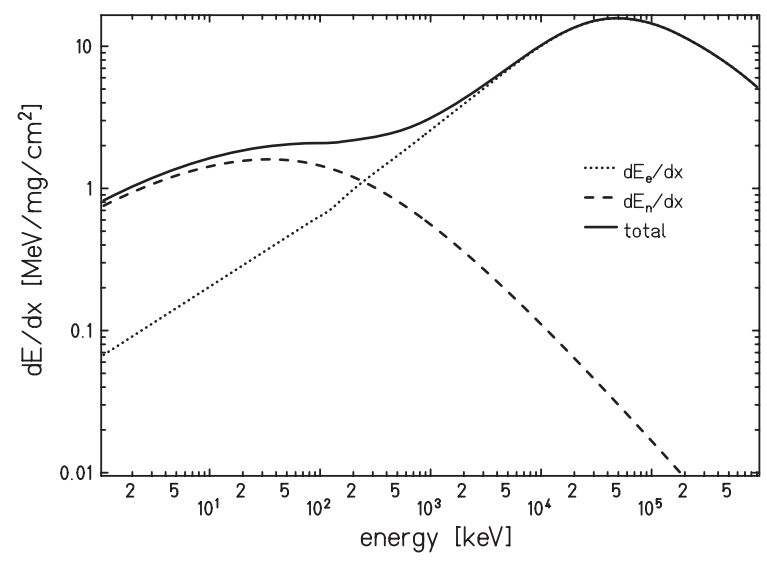

FIG. 1. Potassium ion-energy loss in stainless steel calculated using the SRIM code, the nuclear $\left(d E_{n} / d x\right)$, electronic $\left(d E_{e} / d x\right)$, and total energy losses are displayed. 
sorption coefficients of 9000-14000 molecules/ion were also measured for low-energy $0.025 \mathrm{MeV} / \mathrm{u} \mathrm{K}^{+}$ions incident at grazing angles $\left(\theta=80^{\circ}-88^{\circ}\right)$ onto chemically cleaned stainless steel baked at $220^{\circ} \mathrm{C}$ in a high-vacuum chamber [16].

Enhanced desorption by energetic ions may impact other areas. In magnetic-fusion tokamaks, wall desorption is dominated by physical and chemical sputtering from low-energy ion bombardment [17]. However, some instabilities carry energetic ions to the wall [18], resulting in the more copious electronic desorption. In astrophysics, cosmic ray desorption of gas condensed on dust surfaces can affect gas densities in nebulae, and increase the temperature range over which the normally nonreactive $\mathrm{H}_{2}$ and $\mathrm{CO}$, on iron dust may form $\mathrm{CH}_{4}$ and other prebiotic molecules [19], as are observed in the Orion nebula [20].

All previous molecular desorption yield measurements by heavy-ion beams used fixed ion energy at each facility. In our new studies reported here, molecular desorption from accelerator-type stainless steel is investigated for a range of ion energies at GSI Darmstadt and at Lawrence Livermore National Laboratory (LLNL), plus a range of angles of incidence at LLNL. We found the desorption yield scales with the electronic energy loss $\left(d E_{e} / d x\right)^{n}$, with $n$ between the first and second power, as in many electronic sputtering studies. However, the desorption dependence on $\theta$ is significantly less than $1 / \cos (\theta)$, contrasting with previous studies.

To study the link between molecular desorption and the electronic energy loss of the projectiles in the target, we have measured desorption yields in two energy regimes: (1) A high-energy regime well above the peak of the electronic energy loss, the so-called Bragg-Peak, where $d E_{e} / d x$ decreases with increasing energy. This beam was available at the SIS18 of GSI Darmstadt [21]. (2) A lowenergy regime (70 to $1000 \mathrm{keV}$ with $\mathrm{K}^{+}$ions) for which nuclear slowing dominates below and electronic energy loss dominates above $250 \mathrm{keV}$, as shown in Fig. 1. The opposite slopes and nearly constant sum provide the possibility that the energy scaling of desorption will unambiguously link to one component. The low-energy beam was available at the STS-500 Ion Source Test Stand [22] at LLNL which provided $70-400 \mathrm{keV} \mathrm{K}^{+}$ions. The $\mathrm{HCX}$ at LBNL delivered $\mathrm{K}^{+}$ions at $\sim 1000 \mathrm{keV}[16,23]$.

The measurement technique and experimental setups have been described in detail elsewhere [13,16,24]. Briefly, we determine desorption coefficients by measuring the pressure rise $\Delta p$ in a known volume $V$, or with pumping speed $S$, from which we calculate the number of desorbed molecules. A Faraday cup for the low-energy range, or a current transformer for the high-energy range, measures the beam current transmitted through the entrance aperture, which we integrate to determine the total number of ions $N$ or ion flux $\dot{N}$ incident on a stainless steel target. The ion charge state is known from the accelerator. The effective ion-induced desorption yield $\eta_{\text {eff }}$ (molecules/ion) is given by

$$
\eta_{\mathrm{eff}}=\frac{\Delta p V}{N k_{b} T}
$$

for a single shot of ions (low-energy range) or by

$$
\eta_{\text {eff }}=\frac{\Delta p S}{\dot{N} k_{b} T}
$$

for repetitive ion bombardment (high-energy range), where $k_{b}$ is the Boltzmann constant, and $T$ is the temperature. The data shown here are all derived from pressure rises measured with ion gauges calibrated for nitrogen. With "effective" desorption yield we point out that all measured values from one experimental setup can be compared, since the same systematic errors are valid for all data points. The comparison of desorption yields from different test stands (different conductances, volumes, and vacuum instrumentation like pumps, gauges, and residual gas analyzers), can be done only with extreme care and detailed knowledge, and is not attempted here.

The effective ion-induced desorption yield has been measured for $\mathrm{K}^{+}$ions as a function of the impact angle in the energy range of $68-972 \mathrm{keV}$, as shown in Fig. 2. The experimental setup has been described in Ref. [16].

In this low-energy range experiment the desorption yield increased with rising projectile energy. We measured that grazing incidence of $89^{\circ}$ yields more desorbed molecules than the less grazing incidence of $81.5^{\circ}$, but only by the surprisingly small factor of $\sim 1.5$ as one can see from Fig. 2. If ion-induced desorption were to scale with $[1 / \cos (\theta)]^{m}$, with $m \geq 1$, as in electronic desorption and

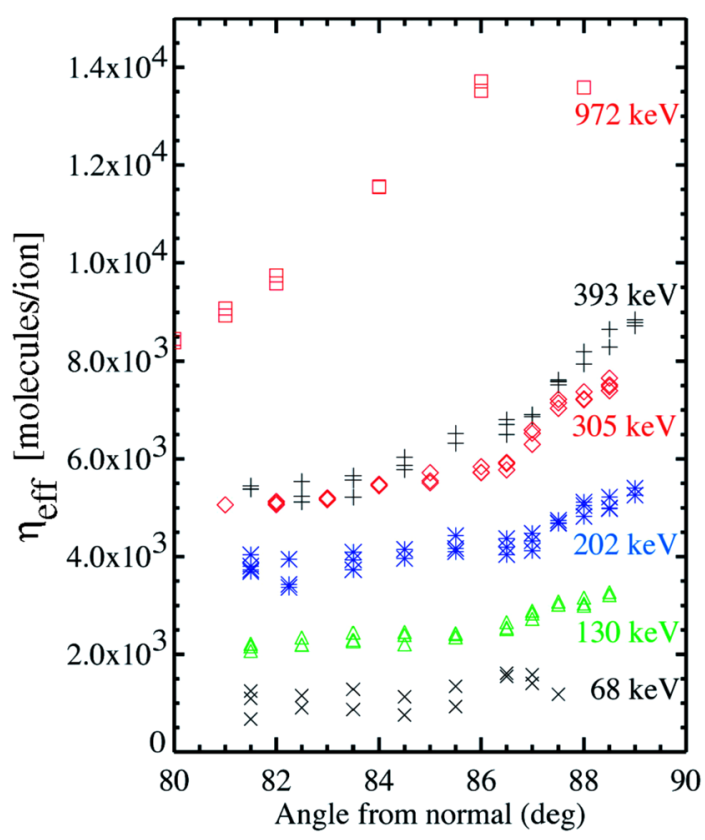

FIG. 2 (color online). Effective desorption yields of potassium ions bombarding unbaked stainless steel at various energies and impact angles. For normal incidence, $\theta=0^{\circ}$. Random variations of \pm 300 are indicated by shot-to-shot scatter. 
sputtering from thick layers, the increase over this range of angles would be a factor of $\geq 8.5$.

A similar angular dependence was previously reported from CERN measurements using $4.2 \mathrm{MeV} / \mathrm{u}$ lead ions incident onto stainless steel, i.e., desorption coefficients between $84.8^{\circ}$ and $89.2^{\circ}$ varied only by a factor of $\sim 2.4$ for $\mathrm{Pb}^{53+}$ and a factor of $\sim 4.5$ for $\mathrm{Pb}^{27+}$ rather than a value of 6.5 expected from a $1 / \cos (\theta)$ variation [13]. The measured desorption coefficients vary as a function of ion impact as follows: for ions onto stainless steel, we obtain $m=$ $0.12 \pm 0.06$ for $\mathrm{Pb}^{53+}$, and $m=0.31 \pm 0.26$ for $\mathrm{Pb}^{27+}$, based on data in Table III of Ref. [13], whereas for electronic sputtering by ions onto insulators one finds $1 \leq m \leq$ $2[5,10]$.

We have not corrected for ion reflection near grazing incidence. The SRIM code [1] predicts that $\sim 70 \%$ of $1 \mathrm{MeV}$ $(0.025 \mathrm{MeV} / \mathrm{u})$ potassium [16] and $\sim 30 \%$ of $4.2 \mathrm{MeV} / \mathrm{u}$ lead ions will reflect, then hit a downstream surface causing additional desorption. A correction for reflections would make desorption even more independent of angle of incidence.

To analyze our data as a function of beam energy and therefore as a function of the total energy loss of the potassium ions in stainless steel, we averaged desorption yields over a narrow range of angles $84.0^{\circ} \leq \theta \leq 85.5^{\circ}$, as plotted in Fig. 3. The choice of angles has only a small effect on the energy scaling as the measured desorption yields for $68-393 \mathrm{keV}$ potassium ions do not significantly change for impact angles between $81.5^{\circ}$ and $85.5^{\circ}$ (see Fig. 2); although the data for $972 \mathrm{keV}$ ions show a greater change. The investigated potassium energy regime is below the Bragg-Peak and one can clearly see from Fig. 3 that the ion-induced desorption yield is increasing with increasing projectile energy following the trend of the electronic energy loss only.

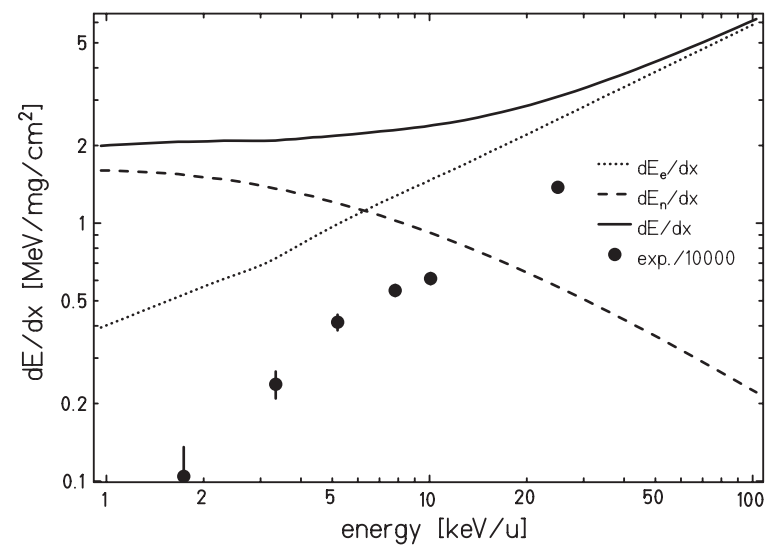

FIG. 3. Nuclear $\left(d E_{n} / d x\right)$ and electronic $\left(d E_{e} / d x\right)$ energy loss for potassium ions in stainless steel calculated with the SRIM code [1], the upper line is the sum of both. The ion desorption yields divided by 10000 were measured for impact angles $(\theta=$ $\left.84.0^{\circ}-85.5^{\circ}\right)$ and are plotted as the points labeled exp./10 000 . The desorption yield error bars $( \pm 300)$ take into account the observed fluctuations in ion beam current and pressure rise which were similar for all low-energy range experiments.
The effective ion-induced desorption yield has been measured in the high-energy regime using $\mathrm{U}^{73+}$ heavy ions with energies of 15,40 , and $100 \mathrm{MeV} / \mathrm{u}$ bombarding a stainless steel target under perpendicular incidence $(\theta=$ $\left.0^{\circ}\right)$. Further details of this work are available elsewhere [24]. A summary of the results obtained is displayed in Fig. 4. Here, we are well above the Bragg-Peak and one can observe that desorption yields again follow the trend of the electronic energy loss, both are decreasing for increasing projectile energy. Both low and high ion-energy desorption yields are plotted in Fig. 5 versus the calculated electronic energy loss $d E_{e} / d x$.

The slopes of the dashed lines in Fig. 5 indicate the power $n$ of the ion-induced desorption scaling with $\left(d E_{e} / d x\right)^{n}$. Both the low-energy $\mathrm{K}^{+}$and the high-energy $\mathrm{U}^{73+}$ results scale roughly with $n=2$. Yields of $10^{3}-10^{4}$ molecules $/ \mathrm{K}^{+}$ion and $10^{2}-10^{3}$ molecules $/ \mathrm{U}^{73+}$ ion have been measured in two different experiments with both showing a dependence of the molecular desorption yield on the electronic energy loss $d E_{e} / d x$.

The measured dependence on $d E_{e} / d x$ limits which models of desorption can be applicable. Elastic thermal spike models yield $n=2$; shock wave models $n=1.5$, and excitation model $n=1$ [25]. With desorption from adsorbed gas layers on the surface of metal, one expects rapid electron cooling by conduction electrons, with inelastic energy absorption by the insulating surface gas layers, for which inelastic thermal spike modes, that allow coupling between electrons and the lattice, may prove more appropriate [25].

Surface properties of stainless steel play a crucial role for the desorption coefficients $[13,26]$. The influence of various surface preparation techniques like vacuum firing at $950{ }^{\circ} \mathrm{C}$, glow discharge cleaning with $\mathrm{Ar}-\mathrm{O}_{2}$ and $\mathrm{He}-\mathrm{O}_{2}$, in situ baking, chemical etching, electropolishing, and coatings with noble metals ( $\mathrm{Au}, \mathrm{Ag}$, Pd) or nonevaporable getters (TiZrV) had been previously studied at CERN and found to affect desorption significantly $[13,26]$. Our results for ions incident on metals show a scaling of desorption

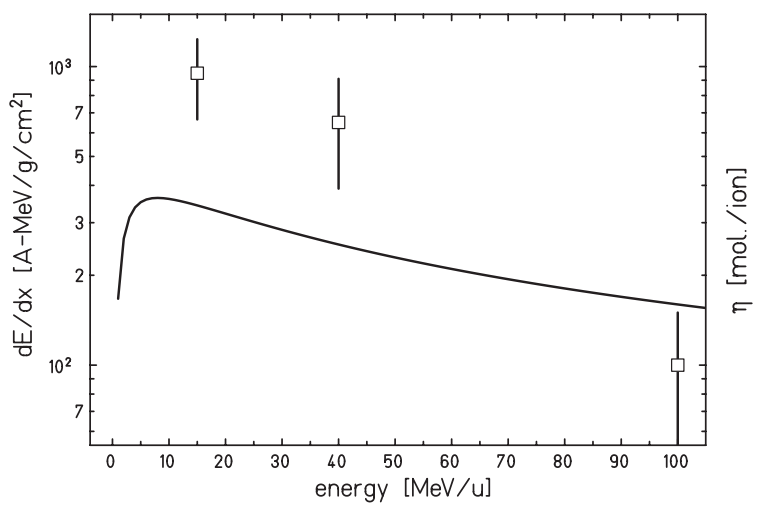

FIG. 4. Desorption yields measured for 15, 40, and $100 \mathrm{MeV} / \mathrm{u} \mathrm{U}^{73+}$ ions impacting perpendicularly onto stainless steel. For comparison, the evolution of the electronic energy loss calculated with SRIM [1] is also shown. 


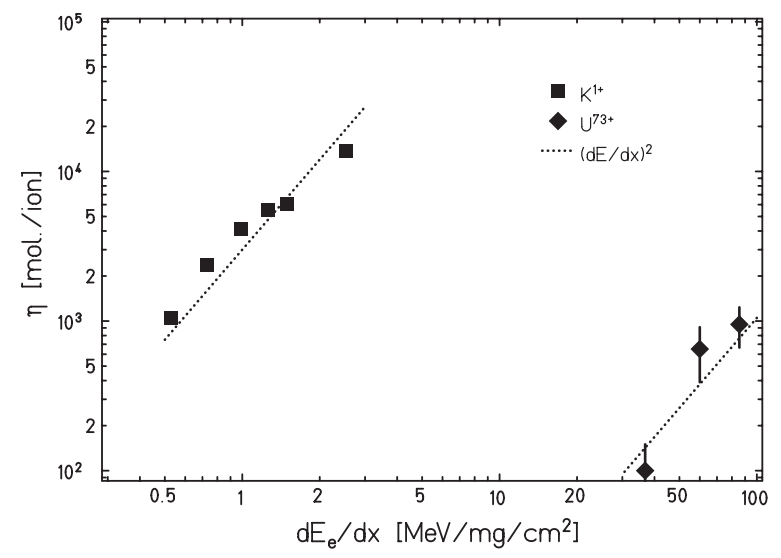

FIG. 5. Measured ion-induced desorption yields versus electronic energy loss of the corresponding ion (potassium and uranium); square points: data for $\mathrm{K}^{+}$ions with energies between 70 and $1000 \mathrm{keV}$ impacting $\left(\theta=84.0^{\circ}-85.5^{\circ}\right)$ onto stainless steel; diamond points: SIS 18 data for $\mathrm{U}^{73+}$ ions with energies 15 , 40, and $100 \mathrm{MeV} / \mathrm{u}$ impacting perpendicularly $\left(\theta=0^{\circ}\right)$ onto stainless steel. The dashed curves indicate the power law ( $n$ value) for the electronic component of $d E / d x$.

with the first to second power of electronic energy loss, similar to that seen for ions incident on frozen gases. However, we observe significantly less yield variation with the angle of incidence, than the $1 / \cos (\theta)$ seen previously for sputter measurements [5,10]. It is important to mention that all electronic energy loss calculations, using the SRIM code [1], were performed for clean stainless steel targets, not taking into account the natural oxide layer or any surface layer contamination like carbon, for example. But exactly this surface layer contamination is predominantly desorbed $\left(\mathrm{H}_{2}, \mathrm{CH}_{4}, \mathrm{CO}, \mathrm{CO}_{2}\right)$ under ion bombardment and scales - as shown - with the electronic energy loss of the projectile in the target.

It is apparent from this work that ion-induced desorption of surface adsorbates is moderated by the energy given to electrons in the target material, but further work is required to determine the details of the mechanism(s). The identification of electronic desorption as the mechanism for gas desorption from metals by energetic heavy ions is a significant step towards understanding ion-induced desorption in accelerators, and more effective mitigation. The data presented here place severe constraints on detailed models of the underlying physical mechanisms and the sources of gas.

A. W. M. thanks T. Schenkel for introducing him to the concept and literature of electronic sputtering, E. Bringa and S. Kucheyev for additional discussions, W. van Breugel and A. G. G. M. Tielens for astrophysical implications, and B.G. Logan for support. Experimental operations at LLNL were ably supported by G. Freeze and R. Hall, and at LBNL by R. Hipple, W. Strelo, R. Beggs, T. Katayanagi, C. Rogers, and E. Romero II. This work was performed under the auspices of the U.S.
Department of Energy by the University of California, Lawrence Livermore and Lawrence Berkeley National Laboratories under Contracts No. W-7405-Eng-48 and No. DE-AC02-05CH11231. H. K., M.C. B., M. B., A. K., E. H., O.B.M., L. W., and E.M. would like to acknowledge the support from the GSI accelerator crew providing an excellent beam.

*Present address: FermiLab, Batavia, IL 60510-0500, USA.

[1] J.F. Ziegler, http://www.srim.org/.

[2] R. E. Johnson, Rev. Mod. Phys. 68, 305 (1996).

[3] W. L. Brown, W. M. Augustyniak, L. J. Lanzerotti, R. E. Johnson, and R. Evatt, Phys. Rev. Lett. 45, 1632 (1980).

[4] W. L. Brown et al., Nucl. Instrum. Methods Phys. Res., Sect. B 14, 392 (1986).

[5] R. E. Johnson et al., Surf. Sci. 179, 187 (1987).

[6] A. Hedin, P. Hakansson, M. Salehpour, and B. U.R. Sundqvist, Phys. Rev. B 35, 7377 (1987).

[7] K. Wien, Radiat. Eff. Defects Solids 109, 137 (1989).

[8] M. Caron et al., Nucl. Instrum. Methods Phys. Res., Sect. B 179, 167 (2001).

[9] M. Caron, H. Rothard, and A. Clouvas, Surf. Sci. 528, 103 (2003).

[10] E. M. Bringa and R. E. Johnson, Nucl. Instrum. Methods Phys. Res., Sect. B 180, 99 (2001).

[11] S. Y. Zhang, Proceedings of the ICFA Workshop on Beam Induced Pressure Risein Rings, http://www.c-ad.bnl.gov/ icfa (2003).

[12] O. Gröbner and R. S. Calder, IEEE Trans. Nucl. Sci. 20, 760 (1973).

[13] E. Mahner et al., Phys. Rev. ST Accel. Beams 6, 013201 (2003).

[14] E. Mahner et al., Phys. Rev. ST Accel. Beams 7, 103202 (2004).

[15] E. Mustafin et al., Nucl. Instrum. Methods Phys. Res., Sect. A 510, 199 (2003).

[16] A. W. Molvik et al., Phys. Rev. ST Accel. Beams 7, 093202 (2004).

[17] G. F. Matthews, J. Nucl. Mater. 337-339, 1 (2005).

[18] W. W. Heidbrink and G. Sadler, Nucl. Fusion 34, 535 (1994).

[19] M. E. Kress and A. G. G. M. Tielens, Meteorit. Planet. Sci. 36, 75 (2001).

[20] G. J. White et al., Astron. Astrophys. 407, 589 (2003).

[21] P. Spiller and G. Franchetti, Nucl. Instrum. Methods Phys. Res., Sect. A 561, 305 (2006).

[22] J. W. Kwan et al., Nucl. Instrum. Methods Phys. Res., Sect. A 544, 134 (2005).

[23] L. R. Prost et al., Phys. Rev. ST Accel. Beams 8, 020101 (2005).

[24] H. Kollmus et al., Proceedings of the 2006 European Particle Accelerator Conference, currently available at http://oraweb.cern.ch/pls/epac06/toc.htm as paper TUPCH174. Subsequently available at JACOW Website.

[25] M. Toulemonde et al., Nucl. Instrum. Methods Phys. Res., Sect. B 212, 346 (2003).

[26] E. Mahner et al., Phys. Rev. ST Accel. Beams 8, 053201 (2005). 